\title{
Rôle du muscle ptérygoidien médial dans les dysfonctions cranio-mandibulaires
}

\author{
Involvement of medial pterygoidis muscle in craniomandibular dysfunctions
}

\author{
STÉPHANIE GAMBADE*, JEAN-MARIE MARTEAU*, PHILIPPE CAIX**
}

\begin{abstract}
RÉSUMÉ
Dans les dysfonctions crânio-mandibulaires, le rôle du muscle ptérygoïdien latéral est bien connu : il intervient notamment, par son insertion sur le vrai-faux disque articulaire de l'ATM. L'implication des muscles masséter et temporal dans l'origine de ces troubles est également bien connue. Il existe peu de données quant à la responsabilité du muscle ptérygoïdien médial dans les désordres crânio-mandibulaires. Ce muscle était initialement adducteur de la mandibule. Comme il a conservé sa spécificité, c'est dans les dysfonctions de la latéralité et de la propulsion que le ptérygoïdien médial intervient.

Les insertions anatomiques du muscle ptérygoïdien médial - de ce fait, la dynamique qu'il exerce sur la mandibule et donc sur l'ATM - amènent à retracer les grandes étapes de l'embryogénèse et de la morphogénèse de ces trois entités anatomiques (ptérygoïdien médial, ATM et mandibule) pour mieux comprendre comment ce muscle intervient dans les dysfonctions cranio-mandibulaires.

L'imagerie des ATM traduit le fait qu'une pathologie musculaire telle l'atteinte du muscle ptérygoïdien médial peut aboutir à une pathologie intra-articulaire. Dans la perte du calage postérieur, le système musculaire est atteint le premier. C'est le ptérygoïdien médial qui va subir des tensions excessives. La souffrance de ce muscle amène la mandibule dans une position anormale. La modification de posture mandibulaire va être à l'origine d'une souffrance des autres muscles élévateurs qui conduira à une modification de la position discale. (Med Buccale Chir Buccale 2006; 12: 73-82).
\end{abstract}

mots clés : ptérygoïdien médial, dysfonctions cranio-mandibulaires, ATM, muscles masticateurs et élévateurs, latéralité, propulsion, embryologie, morphogénèse, anatomie, calage postérieure

\section{SUMMARY}

The role of the medial pterygoidis muscle in cranio-mandibular dysfunctions is well known, in particular, for its insertion on the true-false articular meniscus of the TMJ. The involvement of the masseter and temporal has also been shown in the onset of these disorders. However, few conclusions have been drawn regarding the involvement of the medial pterygoidis muscle in cranio-mandibular disorders. Owing to the specific role that this muscle plays in adduction of the mandible, the medial pterygoidis is involved in laterality and propulsion dysfunctions.

In view of the anatomical insertions of the medial pterygoidis muscle and the dynamics which it exerts on the mandible and the temporo-mandibulare joint, we review the main stages of the embryogenesis and morphogenesis of these three anatomical entities (medial pterygoidis, TMJ and mandible) in order to better understand how this muscle intervenes in cranio-mandibulare dysfunctions.

\footnotetext{
* Faculté de chirurgie dentaire, Bordeaux.

** Université Victor Ségalen - Bordeaux 2 - Laboratoire d’Anatomie Médico-Chirurgicale, 146 rue Léo Saignat 33076 - BORDEAUX Cedex
}

Demande de tirés à part :

Stéphanie Gambade 61 rue Frère 33000 Bordeaux stephbmg@yahoo.fr 
TMJ imaging shows that a muscular pathology involving the attack of the medial pterygoïdis muscle can lead to an intra-articular pathology. In the loss of the posterior shock, the muscular system is involved first. It is the medial pterygoidis which undergoes excessive tension. The strain on the muscle shifts the mandible into an abnormal position. The modification of mandibular posture triggers suffering of the other elevator muscles, in turn leading to a modification of the position of the intervertebral discs. (Med Buccale Chir Buccale 2006; 12: 73-82).

key words : pterygoid muscles, cranio-mandibular disorders, temporo mandibular joint, masticatory and elevatory muscles, laterality, propulsion, embryogenesis, morphogenesis, anatomy, posterior shock

médecine

buccale

chirurgie

buccale

VOL. $12, \mathrm{~N}^{\circ} 2$ 2006

page 74
Le muscle ptérygoïdien médial, ancien muscle ptérygoïdien interne, appartient au groupe des muscles masticateurs (muscles striés crâniofaciaux) comprenant également les muscles masséter, ptérygoïdien latéral et temporal.

Dans les dysfonctions crânio-mandibulaires, le rôle du muscle ptérygoïdien latéral est bien connu : il intervient notamment par son insertion sur le vrai-faux disque articulaire de I'ATM. L'implication des muscles masséter et temporal a également été démontrée dans l'origine de ces troubles. Cependant, il y a peu de données sur la responsabilité du muscle ptérygoïdien médial dans les désordres crânio-mandibulaires.

C'est pourquoi on doit essayer de préciser le rôle de ce muscle qui, se distingue des autres muscles masticateurs, par son individualité, dans la genèse des désordres crânio-mandibulaires.

\section{BREFS RAPPELS PHYLOGÉNÉTIQUES}

Les quatre muscles masticateurs (temporal, masséter, ptérygoïdiens médial et latéral) sont issus, phylogénétiquement, d'une même masse musculaire, située latéralement par rapport à la branche mandibulaire ${ }^{[1]}$. En effet, chez les tétrapodes non-mammaliens, cette unique masse musculaire représentée par le muscle adducteur de la mandibule se divise en deux muscles de part et d'autre de la branche mandibulaire: le temporal (superficiel et latéral) et le ptérygoïdien (plus profond et médial). La fonction masticatoire se compliquant et s'affinant chez les mammifères, le temporal des reptiliens donne, chez les mammaliens, le temporal et le masséter. Le muscle ptérygoïdien se divise en ptérygoïdien médial et ptérygoïdien latéral. La différenciation du muscle ptérygoïdien médial, différenciation initialement phylogénétique puisque ce muscle est issu du muscle adducteur, n'est en fait qu'une différenciation anatomique liée à la fonction qui lui est dévolue. En raison de la spécificité initiale du muscle adducteur de la mandibule, c'est dans les dysfonctions de la latéralité et de la propulsion que ce muscle médial est sollicité.

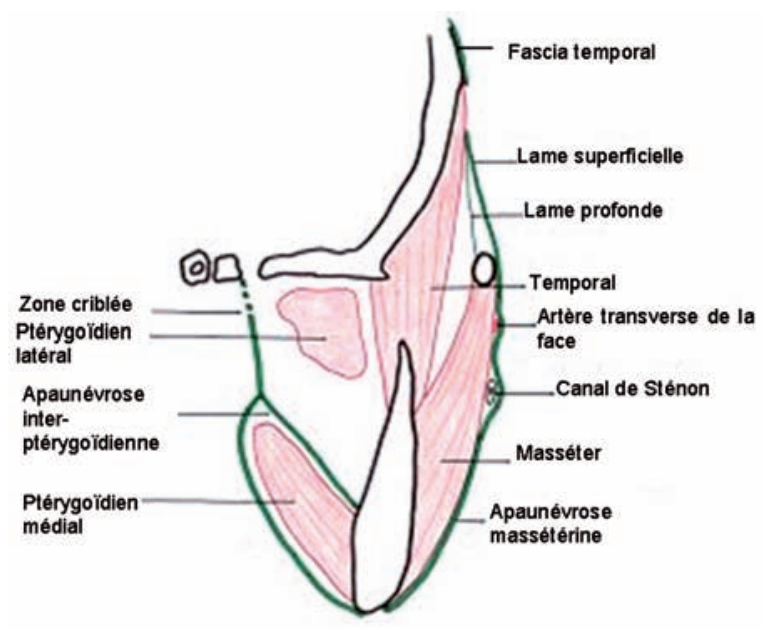

Figure 1 : La sangle ptérygo-massétérine, d'après Rouviere et Delmas [2]

Pterygo-masseterin strap, from Rouviere et Delmas [2] 


\section{EMBRYOGENESE ET MORPHOGE- NESE DU MUSCLE PTERYGOÏDIEN MEDIAL ET DE L'ATM}

\section{Développement du muscle ptérygoïdien médial}

\section{Embryogénèse ${ }^{[3]}$}

Le développement du système musculaire, et donc celui du muscle ptérygoïdien médial, se fait à partir des somites (ou somatomères). Au $21^{\mathrm{e}}$ jour, le phénomène de segmentation aboutit à la disposition de 42 à 44 paires de somites placées de part et d'autre du tube neural et de la chorde dorsale. A la fin de la quatrième semaine, le myotome s'individualise au sein de la paroi interne épaissie de chaque somite. Les cellules du myotome se différencient en cellules fusiformes qui donnent les cellules musculaires souches, ou myoblastes, à l'origine des fibres musculaires striées.

Les muscles masticateurs, dont le ptérygoïdien médial, sont issus d'une partie du premier arc branchial, le bourgeon mandibulaire.

\section{Organogénèse ${ }^{[4]}$}

Le mésenchyme du bourgeon mandibulaire est colonisé par des cellules provenant des crêtes neurales du premier arc branchial. Le muscle ptérygoïdien médial se développe dans ce bourgeon après migration des myoblastes issus du quatrième somite du groupe somitique occipital. Les cellules des crêtes neurales induisent la différenciation des tissus, qui sont à l'origine de la formation des muscles masticateurs.

Il existe un phénomène d'induction réciproque entre :

- les cellules des crêtes neurales qui migrent dans le mésenchyme branchial ;

- le nerf responsable de l'innervation du secteur concerné ;

- la migration et la différenciation des myoblastes du bourgeon mandibulaire du premier arc branchial.

\section{Morphogénèse ${ }^{[4]}$}

L'orientation et la croissance d'un muscle et d'un nerf dans une direction donnée se font grâce à la migration préliminaire des cellules des crêtes neurales. Ces dernières réalisent alors un appel cellulaire qui guide le développement des nerfs et des muscles dans leur parcours.

Le muscle ptérygoïdien médial est initialement à distance des ébauches cartilagineuses qui constitueront secondairement ses insertions ptérygoïdienne, palatine et mandibulaire.

La participation du muscle ptérygoïdien à un mouvement destiné à servir une fonction (la mastication) stimule son développement ; in utéro, c'est le mouvement lié au couple succion-déglutition qui stimule le développement.

Le muscle doit au préalable être mis sous la commande nerveuse responsable du mouvement. Cette commande du nerf se fait par la croissance d'un axone issu du ganglion de Gasser (V3, nerf mandibulaire), en direction de l'ébauche musculaire située dans le bourgeon mandibulaire du premier arc branchial, comme si le muscle ptérygoïdien médial « appelait » son nerf.

\section{Développement de la mandibule et de I'ATM [4]}

Pour mieux comprendre l'importance des insertions anatomiques du muscle ptérygoïdien médial, et de ce fait la dynamique qu'il exerce sur la mandibule et sur l'ATM, on doit retracer les principales étapes de l'embryogénèse et de la morphogénèse pour ces deux entités anatomiques.

\section{Embryogénèse de la mandibule}

Le développement de la mandibule présente sa propre ergonomie. Le bourgeon mandibulaire contient une ébauche cartilagineuse, le cartilage de Meckel, qui précède l'os mandibulaire. Au fur et à mesure que ce cartilage se développe, une condensation des parties molles forme une membrane sur ses faces postéro-inférieures et externes. Puis il se produit une ossification, sans passage par une ébauche cartilagineuse.

Il faut distinguer la « vraie » mandibule de la « fausse » mandibule. La vraie mandibule n'est autre qu'un système en arc dans lequel chemine le V3. C'est la partie génétiquement programmée et sensible à l'hormone de croissance. Le reste des structures osseuses qui constituent la man- médecine

buccale chirurgie buccale

VOL. $12, \mathrm{~N}^{\circ} 2$ 2006

page 75 
médecine

buccale

chirurgie

buccale

VOL. $12, \mathrm{~N}^{\circ} 2$ 2006

page 76 dibule (angle goniaque sur lequel vient s'insérer le muscle ptérygoïdien médial, symphyse, processus coronoïde et condyle) vont progressivement se surajouter. Ces structures sont appelées " os musculaires ", car ils se développent sous l'action des boucles neuro-musculaires de leur voisinage.

\section{Embryogénèse de l'ATM}

Initialement, l'ATM est constitué par un blastème conjonctif comportant d'un côté une surface osseuse correspondant au crâne et, de l'autre, une surface osseuse plus ronde correspondant au futur condyle mandibulaire. Entre ces deux surfaces osseuses, se trouvent deux cavités séparées par un blastème fibreux. Ainsi l'ATM évolue avec ces deux surfaces pseudo-articulaires et l'élément fibreux interposé que représente le futur disque ; il sépare les deux cavités : I'une temporo-discale, l'autre condylo-discale. De part ses origines fibreuses, le disque correspond très probablement à l'extrémité du tendon d'insertion du muscle ptérygoïdien latéral. L'ATM ressemble à une suture crânio-faciale dynamique puisque, dès le soixantième jour in utéro, le fœtus ouvre et ferme la bouche ; l'absence de mouvement articulaire entraîne l'apparition d'un processus d'ankylose. Le muscle ptérygoïdien médial est extra-articulaire même s'il est partie prenante dans les différentes fonctions de l'ATM.

\section{Morphogénèse de l'ATM}

La partie mandibulaire de l'ATM est constituée par un « os musculaire ». En effet, c'est parce que la mandibule « bouge » que l'on a une ATM.

La construction de l'ATM est générée par l'ensemble des boucles neuromusculaires auxquelles elle est dévolue. Chronologiquement les systèmes s'organisent dans l'ordre suivant :

- la succion : dès le trentième ou le quarantième jour, l'embryon avale et aspire le liquide amniotique ;

- la dégutition ;

- la mastication ;

- la phonation.

Ces fonctions - la succion d'abord puis la déglutition - participent à la formation de l'ébauche du muscle ptérygoïdien médial ; par l'orientation de ses fibres, c'est un muscle élévateur qui participe aux mouvements de succion.

Puis le modelage de l'ATM va se faire au cours de la croissance faciale. Jusqu'à six ans, l'étage fronto-orbitaire est prédominant, l'enfant n'a pratiquement pas de mandibule. L'ATM a alors un aspect simiesque, c'est-à-dire qu'elle est plate. A six ans, apparaît la première molaire, ou « dent de six ans ", qui va assurer le calage postérieur lors de l'occlusion dentaire. L'intercuspidation de cette dent avec son antagoniste s'accompagne d'une augmentation de la profondeur de la cavité glénoïde, ce qui va déterminer la future pente condylienne. A partir de sept ans, lors de l'éruption des incisives centrales, le modelage de l'ATM commence réellement. En effet, la mise en place de " la clé incisive ", et la présence d'un cingulum à la face postérieure de chacune des incisives centrales supérieures, vont donner sa forme définitive à l'ATM.

Lors de l'ouverture buccale, le mouvement de l'ATM est guidée par la clé incisive : la face antérieure des incisives mandibulaires glisse sur la face postérieure des incisives supérieures, le long du cingulum. Ce mouvement entraîne le développement du condyle temporal.

\section{Dynamiques histologique et morpholo- gique de l'ATM}

L'ATM est une articulation adaptable si elle reste fonctionnelle. En effet, elle est le siège de phénomènes adaptatifs par remodelage osseux, en fonction des charges résultant de l'action des muscles masticateurs. Au cours de la croissance, une ossification enchondrale et une ossification périostée se développent sur l'apophyse condylienne. De ce fait, elle est composée de tissu osseux haversien compact et de tissu osseux lamellaire.

Il existe une relation entre les formes condyliennes (issues du remodelage condylien) et les types d'usure dentaire (comportement occlusal). Ainsi, l'équilibre entre les forces occlusales travaillantes et non travaillantes correspond à un condyle de forme arrondie.

Le condyle est constitué d'une couche fibrocartilagineuse et d'une couche d'os sous-chondral. Le fibrocartilage se subdivise en trois couches: 
une couche fibreuse (fibres de collagène et fibroblastes), une couche intermédiaire et une couche cartilagineuse. La couche intermédiaire est une couche très riche en cellules indifférenciées ayant une forte capacité de croissance. Selon la charge et la fonction, ces cellules se différencient en :

- fibroblastes pour assurer la réparation de la couche fibreuse ;

- cellules cartilagineuses ;

- ostéoblastes.

\section{Différents types de remodelage}

L'intercuspidie maximale constitue un bouclier protecteur pour l'ATM. Quand nous « serrons les dents, nous ne serrons pas les ATM ». Il y a donc plus de contraintes horizontales que de pressions verticales.

Au cours de la vie, les ATM subissent des transformations par remodelage, en fonction des forces mécaniques qu'elles subissent. Le remodelage est un mécanisme biologique qui sert à maintenir l'équilibre entre la forme de l'ATM et la fonction. Il s'agit d'un phénomène de résorption et d'apposition osseuses de surface en réponse à un stimulus mécanique. Lorsque la fonction est équilibrée, le remodelage est dit « progressif » : l'apposition, c'est-à-dire l'ostéogénèse, est supérieure à la résorption. Lorsqu'il existe une dysfonction, il apparaît un dyssynchronisme dans le remodelage qui devient alors "régressif » ou " périphérique ». Le remodelage régressif se produit lorsque la résorption est supérieure à l'apposition. Le remodelage périphérique a lieu au niveau de la surface articulaire. Cela aboutit à la formation d'ostéophytes, tandis que les condyles mandibulaires prennent une forme en bec de perroquet.

Ce qu'il faut dès maintenant noter, c'est la limite entre l'adaptation et le remodelage physiologiques, et les modifications pathologiques. Lorsque les contraintes deviennent trop répétées, il n'y a plus d'adaptation mais apparition d'une dysfonction. Comme cela a été exposé précédemment, la mise en occlusion des dents de six ans réalise un calage postérieur qui est coordonné par l'ensemble des muscles masticateurs dont le ptérygoïdien médial. Ceci conduit à l'approfondissement de la cavité glénoïde. La présence des dents de six ans et la fonction du muscle ptérygoïdien médial participent au modelage de I'ATM et du disque. Inversement, la perte de ce calage postérieur va conduire à des remaniements internes dans I'ATM : ceci représente donc un co-facteur dans l'apparition des dysfonctions crânio-mandibulaires. En effet, les troubles occclusaux ne sont pas systématiquement à l'origine de dysfonctions crânio-mandibulaires. Des facteurs musculaires, comme ceux liés aux dysfonctions du muscle ptérygoïdien médial, peuvent également y intervenir.

\section{RAPPELS DE L'ANATOMIE DE L'ATM ET DU MUSCLE PTERYGOÏDIEN MEDIAL [5-7]}

Pour mettre en évidence le rôle du muscle ptérygoïdien médial dans les dysfonctions crânio-mandibulaires, il faut prendre en compte quelques éléments anatomiques concernant les différentes pièces mises en jeu.

\section{Anatomie de l'ATM}

L'ATM est une diarthrose constituée :

- de structures osseuses : cavité glénoïde et condyle temporal à la partie supérieure, condyle mandibulaire à la partie inférieure.

- d'éléments cartilagineux : les cartilages articulaires et le disque. Ce dernier vient coiffer en avant le processus condylien et s'oppose à la partie antérieure de la fosse mandibulaire. Son bord postérieur est situé à l'aplomb du condyle mandibulaire et ne le dépasse jamais en arrière. Le disque a de nombreuses attaches sur ses bords, à la fois capsulaires, ligamentaires et musculaires. Il existe un ensemble ligamentaire discocondylien qui assure la coaptation interne du disque avec le processus condylien (structures disco-condyliennes antérieure, postérieure, médiale et latérale).

Lors de l'ouverture buccale, il se produit dans un premier temps une rotation du condyle mandibulaire sous le disque qui initialement ne se déplace pas sous la surface temporale. Puis l'ensemble disco-condylien se déplace vers l'avant en effectuant un mouvement de translation, et passe sous l'éminence temporale. Ainsi, à la fin médecine

buccale chirurgie buccale

VOL. $12, \mathrm{~N}^{\circ} 2$ 2006

page 77 
médecine

buccale

chirurgie

buccale

VOL. $12, \mathrm{~N}^{\circ} 2$ 2006

page 78 de l'ouverture buccale, le condyle mandibulaire est à l'aplomb, voire juste en avant de l'éminence temporale. C'est dans le mouvement de translation qu'intervient le muscle ptérygoïdien médial, tandis que la rotation est sous la dépendance du ptérygoïdien latéral (chef inférieur).

\section{Anatomie descriptive du muscle ptérygoïdien médial}

Le muscle ptérygoïdien médial est un muscle strié, épais, quadrilatère, situé en dedans du muscle ptérygoïdien latéral. Issu de la fosse ptérygoïde, son trajet est oblique en bas, en arrière et en dehors, vers la face interne de l'angle goniaque. Son insertion apicale se fait sur toute la fosse ptérygoïde, excepté sur sa paroi interne et sur la fossette scaphoïde. Le corps charnu du muscle se termine sur la face médiale de l'angle de la mandibule, au niveau de la tubérosité ptérygoïdienne. II est innervé par une branche du nerf mandibulaire (V3)

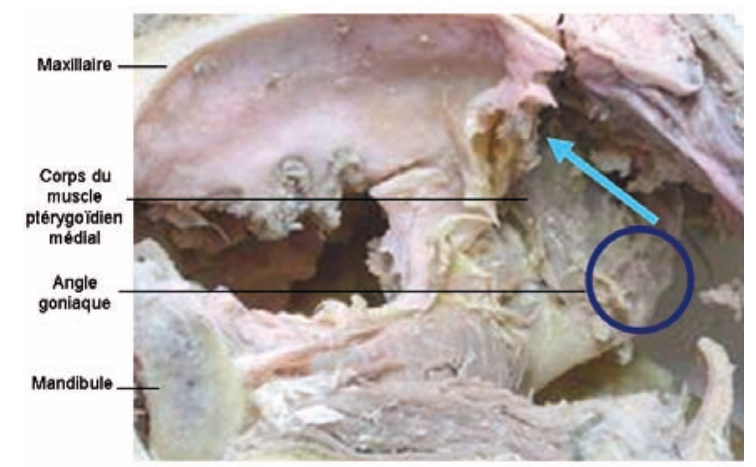

Figure 2 : Insertion caudale du muscle ptérygoïdien médial Caudal insertion of medial pterigoidis muscle

S'il se contracte bilatéralement, il est élévateur de la mandibule. S'il se contracte unilatéralement, il est légèrement diducteur par la disposition oblique de ses fibres. Le muscle ptérygoïdien médial est également propulseur de la mandibule par son orientation de haut en bas et d'arrière en avant. Ainsi, le muscle ptérygoïdien médial intervient dans la phase d'écrasement pendant la mastication.

\section{IMAGERIE DE L'ATM ET DU MUSCLE PTERYGOÏDIEN MEDIAL}

\section{Exploration du muscle ptérygoïdien médial ${ }^{[8]}$}

L'I.R.M permet l'étude du muscle ptérygoïdien médial. Du fait de son orientation oblique en bas et en arrière et en dehors vers la face interne de l'angle goniaque, il est difficile d'obtenir des clichés qui mettent en évidence le muscle ptérygoïdien médial. Les coupes coronales réalisées très postérieurement par rapport au condyle mettent en évidence l'insertion goniaque du muscle avec sa participation à la sangle ptérygo-masséterine. De plus ces coupes doivent être faites selon l'obliquité du muscle.

Les coupes sagittales réalisées en position très médiale mettent en évidence l'insertion apicale du muscle sur les apophyses ptérygoïdiennes. II faudra que le radiologue réalise des coupes sagittales obliques.

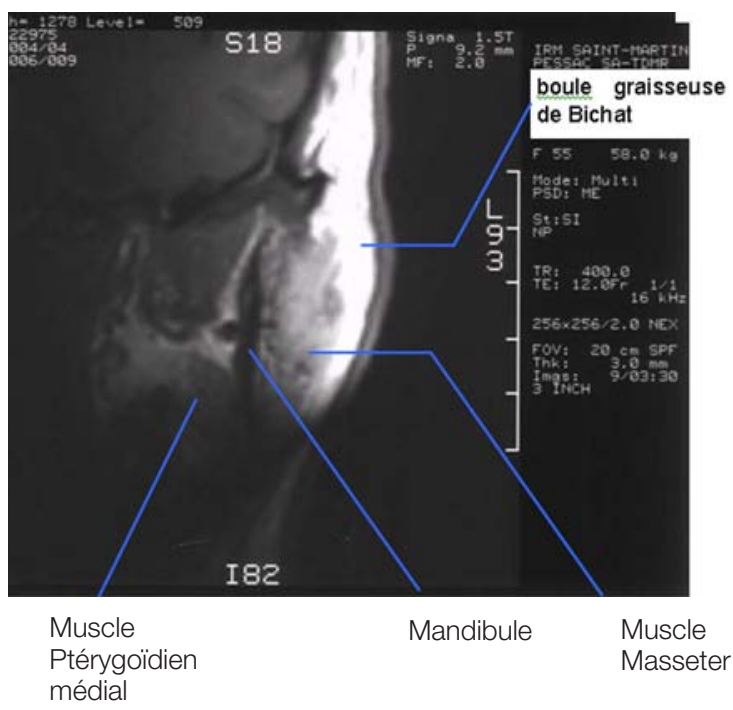

Figure 3 : Coupe coronale passant par la branche montante mandibulaire

$M R I$ of temporomandibular joint with mouth closed. Sagittal view

L'imagerie est en premier lieu utilisée pour mettre en évidence la présence de désordres intra-articulaires dans l'ATM. L'étiologie de ces désordres apparaît rarement sur les clichés. De ce fait, l'imagerie du muscle ptérygoïdien médial n’a que peu d'intérêt pour le diagnostic d'une dysfonction crâ- 
nio-mandibulaire. Le seul avantage qu'apporte l'exploration du muscle ptérygoïdien médial est d'ordre anatomique. Son rôle étiologique est recherché lors de l'examen clinique par sa palpation et la mise en évidence de points douloureux.

\section{Imagerie de l'ATM}

Elle est indispensable lorsqu'il existe un doute sur la nature mécanique de l'atteinte discale, pour exclure une autre cause (musculaire, osseuse, psychique), ou pour déterminer le moment exact de la réduction lors d'une luxation réductible.

\section{Tomographie}

Cet examen peu coûteux, faiblement irradiant, permet une analyse intéressante des structures suivantes :

- la pente condylienne ;

- la morphologie du condyle ;

- l'aspect de l'interligne articulaire.

Les pathologies osseuses peuvent être appréhendées par la présence d'une abrasion et d'ostéophytes.

L'anomalie du disque articulaire ne se fait pas directement, mais par défaut.

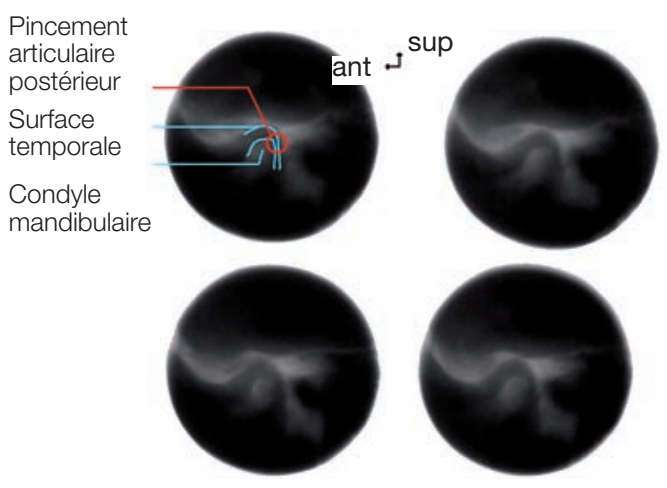

Figure 4 : Tomographie de l'ATM gauche sans gouttière CT scan of temporomandibular joint without splint

\section{Examen tomodensitométrique}

Cet examen est moins irradiant mais plus coûteux que la tomographie ; c'est pourquoi il vient en deuxième intention. II permet une analyse des différentes densités osseuses dans le compartiment articulaire et la multiplication des plans de coupe sans irradiation supplémentaire. II est cependant difficile de bien localiser le disque articulaire.

\section{Arthrographie}

Ses avantages sont la description anatomique des différents composants de l'ATM et la possibilité de réaliser une étude dynamique. La localisation du disque articulaire est mise en évidence par défaut.

\section{IRM}

Cet examen est la seule technique qui permette d'évaluer dans le même temps l'os médullaire et les tissus mous, et de contrôler l'ouverture buccale. II présente un aspect dynamique : six séquences successives de neuf images, à des moments différents de l'ouverture buccale, sont réalisées dans le plan sagittal.

Il permet d'évaluer les déplacements discaux chez les patients porteurs d'un dérangement interne, d'une laxité de l'ATM ou au contraire d'un disque fixé.

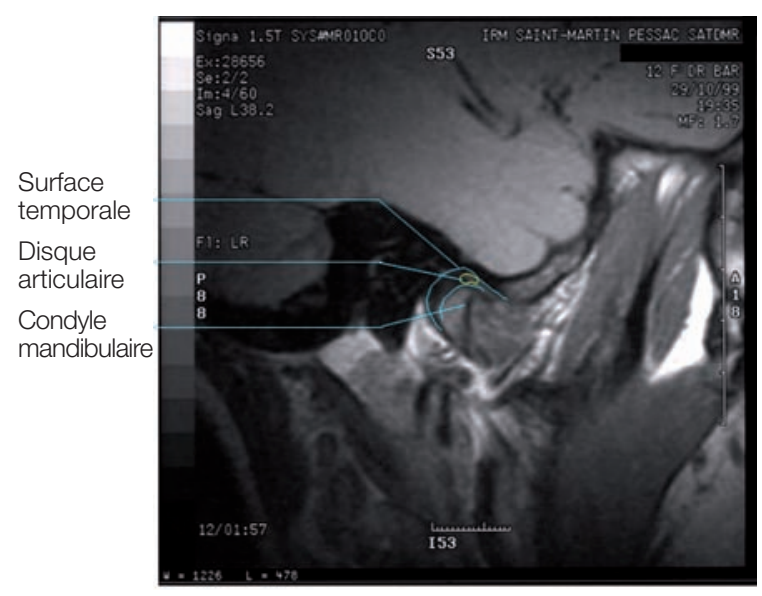

médecine

buccale chirurgie buccale

VOL. $12, \mathrm{~N}^{\circ} 2$ 2006

page 79

Figure 5 : IRM de l'ATM bouche fermée. Coupe sagittale MRI of temporomandibular joint with mouth closed. Sagittal view

En réalité, l'imagerie des ATM comporte globalement deux parties :

- l'imagerie centrée sur le squelette ;

- l'imagerie centrée sur le disque.

L'imagerie du disque est représentée par l'arthrographie et l'IRM. Ces deux examens mettent en relation le disque articulaire et les structures anatomiques.

L'imagerie squelettique, représentée par l'examen tomodensitométrique et la tomographie spi- 
ralée, permet de visualiser les structures osseuses et de déterminer la valeur de l'interligne articulaire. Ainsi, un pincement articulaire peut être mis en évidence et, de ce fait, une malposition du disque.

L'imagerie des ATM démontre qu'une pathologie musculaire - en particulier dans cette étude sur l'implication du muscle ptérygoïdien médial dans les dysfonctions crânio-mandibulaires - peut aboutir à une pathologie intra-articulaire. II faut évaluer l'importance de l'atteinte de l'ATM afin d'estimer s'il existe une éventuelle corrélation entre l'atteinte du muscle et celle de l'articulation. II devient ainsi possible de constater les conséquences intra-articulaires de l'atteinte des mouvements de propulsion et de diduction.

médecine

buccale

chirurgie buccale

VOL. $12, \mathrm{~N}^{\circ} 2$ 2006

page 80

\section{IMPLICATION DU MUSCLE PTERYGOÏDIEN MEDIAL DANS LES DYSFONCTIONS CRANIO-MANDIBULAIRES ${ }^{[10]}$}

\section{Perte du calage postérieur}

La perte du calage postérieur correspond à la perte de la "clé molaire ", c'est-à-dire la disparition de l'articulation ou du contact entre la première molaire maxillaire avec la première molaire mandibulaire. La perte du calage postérieur, dit aussi « calage molaire ", devient effective lorsqu'il y a disparition d'une ou plusieurs molaires. Elle peut être uni ou bilatérale, uni ou bimaxillaire. L'édentement peut être encastré ou terminal ; il peut intéresser les molaires et les prémolaires, voire les dents antérieures, ou être complet. Quelquefois, le calage postérieur peut être perdu alors que les molaires sont encore présentes sur l'arcade : par exemple par usure excessive de la face occlusale lors d'un bruxisme sévère.

\section{Conséquence de la perte du calage postérieur}

\section{Perte des repères desmodontaux}

Il existe des récepteurs parodontaux dont la présence est directement liée à celle de l'organe dentaire. Ces récepteurs n'interviennent que lorsqu'il y a un contact dentaire. L'absence de calage pos- térieur s'accompagne d'une modification des informations fournies par les récepteurs parodontaux : la mandibule adopte une position différente car elle cherche à établir de nouveaux contacts entre les dents maxillaires et mandibulaires restantes. De plus, ce sont des propriocepteurs situés au niveau des tendons musculaires et des capsules articulaires qui vont suppléer les organes disparus.

\section{Instabilité occlusale}

L'établissement de nouveaux contacts dentodentaires se traduit par :

- une propulsion mandibulaire ;

- des mouvements latéraux de diduction;

- une diminution de la dimension verticale d'occlusion (DVO) postérieure et une compression temporo-mandibulaire par l'ascension du condyle mandibulaire dans la cavité articulaire.

La conservation de la DVO postérieure ou de la dimension verticale faciale postérieure assure la protection de l'ATM.

\section{Tension musculaire du ptérygoïdien médial}

La perte du calage postérieur met immédiatement en action les muscles ptérygoïdiens médiaux qui assurent la propulsion, la diduction et l'élévation mandibulaires. Cependant, leur action se fait ici dans le cadre d'une parafonction puisqu'elle vient compenser une instabilité occlusale. L'instabilité occlusale augmente les contractions musculaires. Lors de la perte du calage postérieur, l'activité électromyographique du muscle ptérygoïdien médial augmente avec apparition de cycles fonctionnels irréguliers.

Le circuit de rétro-action propriocetive initié à partir des récepteurs parondontaux est perturbé par la perte de contact dans le secteur molaire. Si la capacité de coordination et d'adaptation du système neuro-musculaire se trouve dépassée, cela peut alors engendrer des troubles dysfonctionnels.

L'ensemble de ces facteurs contribue à la mise en place d'une tension dysfonctionnelle du muscle ptérygoïdien médial, entraînant en retour une sur-stimulation des récepteurs parodontaux lors de la position de compensation. 


\section{Compression temporo-mandibulaire}

La dimension verticale est maintenue par « l'articulation inter-molaire », et par le jeu de l'équilibre musculaire qui en découle. La perte du calage postérieur est responsable d'une perte de la dimension verticale postérieure ce qui se traduit, dans le compartiment articulaire, par une compression temporo-mandibulaire consécutive à l'ascension du condyle mandibulaire. L'ascension du condyle est la conséquence de l'action élévatrice du muscle ptérygoïdien médial. Lorsque les forces dépassent la capacité de remodelage et d'adaptation de l'ATM, l'équilibre entre forme et fonction n'existe plus. Une pathologie peut alors s'installer et évoluer vers une dégénérescence (dégénérescence précoce). Lors de la perte du calage postérieur, les forces excessives qui s'exercent sur l'ATM ont une double origine : la surtension du muscle ptérygoïdien médial (entre autres) et les conséquences de la suractivité de ce muscle ; cette suractivité entraîne un proglissement mandibulaire antérieur, donc le déplacement de la tête du condyle vers l'avant et vers le haut. Cette compression temporo-mandibulaire peut se surajouter à des troubles dysfonctionnels existants ou ayant laissé des séquelles.

\section{Compression temporo-mandibulaire et remodelage articulaire}

La perte des dents influence le remodelage articulaire, responsable du changement de forme de l'éminence articulaire et du condyle mandibulaire : en conséquence " nous avons I'ATM de nos dents ». La perte du calage postérieur peut entraîner des destructions tissulaires dans les ATM.

Les corticales temporales et surtout mandibulaires sont alors irrégulières, minces, avec des zones de résorption et de discontinuité, faisant communiquer le fibrocartilage avec de larges espaces médullaires. Le fibrocartilage augmente d'épaisseur et sa structure habituelle en trois couches n'existe plus. Des zones de dégénérescence apparaissent.

Le disque présente un grand nombre de cellules chondroïdes ; la chondrification représente un signe d'usure. Les feuillets synoviaux subissent une dégénérescence fibreuse. Le frein discal postérieur est désorganisé. Dans les formes sévères, on observe une détérioration progressive de tous les composants articulaires et une incoordination des surfaces articulaires.

\section{CONCLUSION}

Phylogénétiquement, le système masticateur forme une grande unité musculaire. Les muscles masséter et ptérygoïdien médial ne constituent qu'une seule et même masse musculaire, soutenue par la sangle ptérygo-masséterine qui est rompue dans toutes les ostéotomies mandibulaires. Lorsqu'un élément de cette unité masticatrice présente une défaillance, le système intégral est déséquilibré dans son intégralité.

Dans une dysfonction comme la perte du calage molaire, le système musculaire est le premier atteint. A la fin de la période d'adaptabilité musculaire, le système masticateur évolue vers une dysfonction articulaire, la luxation discale étant la plus courante.

Le muscle ptérygoïdien médial, dont l'atteinte ne peut être objectivée que par l'examen clinique, n'a aucune insertion anatomique sur le « vrai-faux disque » de l'ATM. Néanmoins, en présence d'une compression temporo-mandibulaire, sa contracture permanente (ptérygoïdite) rompt l'équilibre du système musculaire masticateur élévateur. II peut donc être indirectement à l'origine de troubles intra-articulaires tels que les luxations discales réductibles ou irréductibles, via l'atteinte du masseter, du temporal et du ptérygoïdien latéral. médecine

buccale chirurgie buccale

VOL. $12, \mathrm{~N}^{\circ} 2$ 2006

page 81 


\section{RÉFÉRENCES}

1 - Beaumont A, Cassier P. Les Cordées, anatomie compaRÉE DES vertébrés (pp 366-373). In Biologie animale, Dunod Université, Bordas, Paris 1982.

2 - Rouviere H, Delmas A. Anatomie humaine. descriptive, topographique et fonctionnelle. Tome 1. Tête et cou (pp166-168). Masson, Paris 2002.

3 - Couly G. Développement céphalique. Embryologie. Croissance. Pathologie (pp 11-45, 55-57). Edition CdP, Paris 1991.

4 - CAIX P. L'articulation temporo-mandibulaire et son vraifaux disque. Approche morphologique, fonctionnelle, clinique et chirurgicale. Thèse de Doctorat d'Etat en biologie humaine - Amiens 1991.

5 - PUTZ R, PABST R. Sobotta. Atlas d'anatomie humaine. Tome 1. Tête, cou, membre supérieur (pp 71). Editions médicales internationales, Cachan 2000.
6 - ROUVIERE H. Anatomie humaine descriptive et topographique. Masson, Paris 1962.

7 - Bouchet A, Cuilleret J. Anatomie topographique, descriptive et fonctionnelle. 1 Le système nerveux central, la face, la tête et les organes des sens (pp 353-384). SIMEP, Paris 1982.

8 - MARSOT-DUPUCH K. Savoir faire en imagerie O.R.L et cervico-faciale. (pp 37-46). Sauramps médical, Montpellier 2002.

9 - BUthiAu D, Dichamp J, GOUDOT P. IRM de l'articulation temporo-mandibulaire (pp15-53). Vigot, Paris 1994.

10 - HaRTMANn F, CUCCHI G. Les dysfonctions cranio-mandibulaires (SADAM). Nouvelles implications médicales (pp 1-4, 5-6, 17-24, 82-108). Springer-Verlag, Paris 1993. médecine

buccale

chirurgie

buccale

VOL. $12, \mathrm{~N}^{\circ} 2$ 2006 